\title{
Contact force mapping during catheter ablation for atrial fibrillation: procedural data and one-year follow-up
}

Alexander Wutzler, Martin Huemer, Abdul Shokor Parwani, Florian Blaschke, Wilhelm Haverkamp, Leif-Hendrik Boldt

Charité - Universitätsmedizin, Berlin, Germany

Submitted: 22 May 2013

Accepted: 21 July 2013

Arch Med Sci 2014; 10, 2: 266-272

DOI: $10.5114 /$ aoms.2014.42578

Copyright ๑ 2014 Termedia \& Banach

\begin{abstract}
Introduction: Pulmonary vein isolation (PVI) is the state-of-the-art treatment of atrial fibrillation (AF). Pulmonary vein reconnection is one of the main mechanisms of $\mathrm{AF}$ recurrence after ablation. Catheter-tissue contact is essential for effective ablation lesions. The aim of this study was to evaluate the impact of catheter contact monitoring during PVI on AF recurrence rate. Material and methods: One hundred and forty-three patients who underwent PVI were analysed. In 31 patients, PVI was performed by monitoring the catheter-tissue contact with a contact force (CF) sensing catheter. One hundred and twelve patients in whom conventional PVI was performed without CF information served as the control group. Procedural data and recurrence rate within 12-month follow-up were compared.

Results: A significant reduction in procedure duration was seen in the CF mapping group (128.4 \pm 29 min vs. $157.7 \pm 30.8 \mathrm{~min}, p=0.001)$. Complete pulmonary vein isolation was achieved in $100 \%$ of the patients. Rate of AF recurrence within 12 months after ablation was significantly lower in the contact force group (16.1\%) when compared to the standard ablation group $(36.6 \%)(p=0.031)$.

Conclusions: Pulmonary vein isolation with the use of contact force information results in a shorter procedure duration and a lower rate of AF recurrence after 12 months compared to conventional PVI without this information. Catheter-tissue contact monitoring may have a beneficial effect on mid-term and long-term results of PVI procedures.
\end{abstract}

Key words: catheter-tissue contact, atrial fibrillation, pulmonary vein isolation.

\section{Introduction}

Radiofrequency (RF) catheter isolation of the pulmonary veins (PV) has become a standard and potentially curative treatment of symptomatic drug refractory atrial fibrillation (AF) [1]. Atrial fibrillation ablation procedures have been shown to be safe and effective in a large number of cases worldwide [1]. However, recurrence rates of AF after catheter ablation are still considerably high and repeat procedures are frequently necessary in these patients $[1,2]$. Pulmonary vein reconduction due to ineffective ablation lesions has been identified as a main cause of AF recurrence [3] and catheter-tissue contact is essential for effective ab-

\author{
Corresponding author: \\ Alexander Wutzler MD, MA \\ Charité - Universitätsmedizin \\ Augustenburger Platz 1 \\ 13353 Berlin, Germany \\ Phone: +4930450565411 \\ E-mail: \\ alexander.wutzler@charite.de
}


lation lesions [4-7]. On the other hand, accuracy of the lesion and limitation of contact force are important matters in order to avoid any perforation of the left atrial (LA) wall or damage to adjacent structures such as the oesophagus or the phrenic nerve $[8,9]$. New technological approaches have recently become available to provide force control. Catheter-tissue contact force (CF) can be measured at the catheter tip with fibreoptic [6] or magnetic [10] sensors or an impedance calculation algorithm [11].

The feasibility and efficacy of fibreoptic CF measurement with an open irrigated ablation catheter (TactiCath; Endosense SA, Meyrin/Geneva, Switzerland) has been studied in animals [4] and in humans $[6,12,13]$. It has been shown in human studies, where the physician was blinded to any contact force information, that low contact force was associated with $\mathrm{PV}$ reconnection 3 months after ablation [12] and was a predictor for $A F$ recurrence 12 months after ablation [13].

However, no study that directly compares AF ablation with the use of fibreoptic force control with conventional RF ablation is available. Only a few studies report intraprocedural results and in most studies the electrophysiologist was blinded as to the CF information. It is reasonable and has been shown in a few studies $[10,11]$ that CF information is useful to increase efficacy (and presumably safety) of the procedure. Yet, it is unclear what impact the technology has on procedure duration, efficacy and safety in daily practice. Furthermore, mid-term and long-term results of ablation procedures performed with contact force information are still rare.

The purpose of our study was to evaluate the procedural characteristics of catheter ablation for atrial fibrillation with and without the use of the TactiCath system. We used a non-randomised, single centre study with data from the local prospective atrial fibrillation ablation registry.

\section{Material and methods}

\section{Study population}

Consecutive patients presented to our centre for catheter ablation of drug refractory symptomatic atrial fibrillation were enrolled between 2010 and 2012. Thirty-one patients underwent catheter ablation with the use of TactiCath. Patients $(n=112)$ who underwent catheter ablation without the use of contact force mapping between 2010 and 2012 served as a control group. Repeat procedures and cases where alternative ablation strategies (linear lesions, ablation of complex fractionated atrial electrograms) or alternative techniques [14] (cryoablation, "single shot" devices, robotic ablation) were used were excluded from the study. Patients with left ventricular ejection fraction (LVEF) $<45 \%$ or status post-surgical valve replacement were also excluded.

\section{Ablation procedures}

Procedures were performed as described previously [15]. Patients were in a fasting state, and transoesophageal echocardiography was performed prior to the ablation to rule out a left atrial thrombus.

After double transseptal puncture, an open irrigated ablation catheter (CoolPath, IBI/St. Jude Medical, St. Paul, MN, USA, $n=112$ ) or an open irrigated ablation catheter with CF mapping capabilities (TactiCath; Endosense SA, Meyrin/Geneva, Switzerland, $n=31$ ) was positioned in the left atrium (LA). A circular mapping catheter (Optima, IBI/ St. Jude Medical, Connecticut, USA) was positioned at the PV ostia for diagnosis of PV connection and confirmation of electrical PV disconnection after ablation. Circumferential pulmonary vein isolation was performed with a 3D-Mapping-System (Ensite NavX, St. Jude Medical) and a Stockert 70 RF generator (Biosense Webster) with a maximal temperature of $43^{\circ} \mathrm{C}$ and a maximum power of $35 \mathrm{~W}$. Complete isolation of the pulmonary veins was the procedural endpoint and was verified by bidirectional block with the circular and the ablation catheter. A temperature probe for monitoring of the oesophageal temperature was used in all cases.

In procedures performed with the TactiCath, physicians were able to view CF information in real time throughout the procedure. Amplitude and orientation of real-time CF (recorded every $100 \mathrm{~ms}$ ), average force (Fav in grams-force, g), and force-time integral (FTI = real-time force $\times$ ablation time, $g \times s$ ) were measured and displayed on an additional screen [4].

Procedures were performed by physicians with at least 4 years experience in interventional electrophysiology, and who perform > 100 PVIs a year. All patients gave written informed consent. The study was approved by the local ethics committee of the Charité Universitätsmedizin Berlin.

\section{Follow-up}

Patients were followed up in the outpatient clinic of our institution. Follow-up visits including 12-lead and 7-day Holter ECG were scheduled 12 months after discharge and performed additionally on non-scheduled visits if patients presented with any symptoms suggestive of AF. Episodes of AF or other atrial arrhythmias lasting longer than $30 \mathrm{~s}$ were considered for analysis. Episodes that occurred within 12 months after ablation with a blanking period of the first 3 months 
after ablation were considered to indicate a recurrence of AF. Antiarrhythmic drugs were continued for the first 3 months after ablation and then discontinued. Patients were followed up over 1 year and procedural complications and outcomes were compared.

\section{Statistical analysis}

Categorical data were expressed as proportions and analysed using the $\chi^{2}$ test. Continuous variables were presented as mean \pm standard deviation (SD). Comparisons were made using the 2-sample $t$-test for normally distributed data and using the Wilcoxon rank sum test for skewed data. All analyses were performed using SPSS software version 20.0 (SPSS Inc., Chicago, IL, USA). A $p$ value of $<0.05$ was considered to be statistically significant.

\section{Results}

A total of 143 patients who underwent catheter ablation for symptomatic drug refractory atrial fibrillation were analysed. Thirty-one patients were ablated with the use of the Tacticath and 112 patients were ablated with standard mapping and ablation technique without the use of contact force information. Patient characteristics are list- ed in Table I. There were no statistically significant differences seen between the groups at baseline.

Results of procedural data are listed in Table II. Procedure duration was significantly shorter in the contact force group (128.4 \pm 29 min vs. $157.7 \pm 30.8$ $\min , p=0.001)$. There were no significant differences observed in ablation time, total ablation energy or fluoroscopy time, although all were reduced in the contact force group (Table II). In the contact force group, the average force was $26.8 \pm 10.7 \mathrm{~g}$ and the force-time integral was $1449.4 \pm 415$ gs.

Complete pulmonary vein isolation as the acute procedural endpoint was achieved in all patients. No steam pops were recognized in any of the groups. One major complication (pericardial effusion) and three minor complications (femoral haematoma) occurred in the standard mapping group, whereas one minor complication (femoral haematoma) was seen in the contact force group. Pericardial effusion was successfully treated with pericardial puncture and drainage.

Haemodynamic and oxygenation changes during anaesthesia stayed within the ranges of previous studies [15]. No case of severe or prolonged haemodynamic or respiratory instability due to sedation occurred. Baseline standard blood test showed no significant differences between

Table I. Patients' characteristics and medication

\begin{tabular}{|c|c|c|c|}
\hline Parameter & $\begin{array}{l}\text { Contact force } \\
\qquad(n=31)\end{array}$ & $\begin{array}{l}\text { Standard mapping } \\
\qquad(n=112)\end{array}$ & Value of $p$ \\
\hline Age, mean \pm SD [years] & $59.8 \pm 10.9$ & $60.9 \pm 10.2$ & 0.61 \\
\hline Gender, male, $n(\%)$ & $21(67.7)$ & $71(63.4)$ & 0.66 \\
\hline Paroxysmal AF, $n(\%)$ & $19(61.3)$ & $85(75.9)$ & 0.11 \\
\hline LAD, mean \pm SD [mm] & $41.5 \pm 6.1$ & $42.4 \pm 6.7$ & 0.59 \\
\hline LVEF, mean \pm SD $(\%)$ & $56.8 \pm 4.9$ & $55.6 \pm 3.1$ & 0.068 \\
\hline Structural heart disease, $n(\%)$ & $7(22.6)$ & $28(25)$ & 0.78 \\
\hline Hypertension, $n$ (\%) & $20(64.5)$ & $58(51.8)$ & 0.21 \\
\hline CAD, $n(\%)$ & $7(22.6)$ & $16(14.3)$ & 0.27 \\
\hline Diabetes mellitus, $n$ (\%) & $3(9.7)$ & $10(8.9)$ & 0.91 \\
\hline $\mathrm{BMI}$, mean $\pm \mathrm{SD}\left[\mathrm{kg} / \mathrm{m}^{2}\right]$ & $27.1 \pm 3.3$ & $27.9 \pm 4.5$ & 0.64 \\
\hline \multicolumn{4}{|l|}{ Baseline medication: } \\
\hline Amiodarone, $n(\%)$ & $2(6.5)$ & $4(3.6)$ & 0.48 \\
\hline$\beta$-Blocker, $n$ (\%) & $28(90.3)$ & $99(88.3)$ & 0.76 \\
\hline Digitoxin, $n(\%)$ & $0(0)$ & $3(2.7)$ & 0.51 \\
\hline ACEI, $n(\%)$ & $15(48.4)$ & $71(63.4)$ & 0.34 \\
\hline ARB, $n(\%)$ & $2(6.5)$ & $13(11.6)$ & 0.87 \\
\hline $\mathrm{CCB}, n(\%)$ & $2(6.5)$ & $4(3.6)$ & 0.095 \\
\hline Statin, $n(\%)$ & $8(25.8)$ & $36(32.1)$ & 0.58 \\
\hline Diuretics, $n(\%)$ & $2(6.5)$ & $13(11.6)$ & 0.87 \\
\hline Aspirin, $n(\%)$ & $18(58.1)$ & $49(43.8)$ & 0.21 \\
\hline Oral anticoagulants, $n(\%)$ & $31(100)$ & $112(100)$ & - \\
\hline
\end{tabular}

$A C E I$ - angiotensin-converting enzyme inhibitor, $A F$ - atrial fibrillation, $A R B$ - angiotensin receptor blocker, $B M I-b o d y$ mass index, $C A D$ - coronary artery disease, $C C B$ - calcium channel blocker, $L A D$ - left atrial diameter, $L V E F$ - left ventricular ejection fraction 
Table II. Procedural data and follow-up results

\begin{tabular}{|lccc|}
\hline Parameter & $\begin{array}{c}\text { Contact force } \\
(n=31)\end{array}$ & $\begin{array}{c}\text { Standard mapping } \\
(n=112)\end{array}$ & Value of $p$ \\
\hline Ablation time, mean \pm SD [min] & $38.6 \pm 12.7$ & $45.2 \pm 16.5$ & 0.13 \\
\hline Procedure duration, mean \pm SD [min] & $128.4 \pm 29$ & $157.7 \pm 30.8$ & $0.001^{*}$ \\
\hline Total energy delivered, mean \pm SD [Ws] & $71964.4 \pm 17894.8$ & $78579.3 \pm 45534$ & 0.9 \\
\hline Average force, mean \pm SD [g] & $26.8 \pm 10.7$ & - & - \\
\hline Force-time integral, mean \pm SD [gs] & $1449.4 \pm 415$ & - & - \\
\hline Fluoroscopy time, mean \pm SD [min] & $39.7 \pm 11.3$ & $43.8 \pm 14.5$ & 0.5 \\
\hline Major complications, $n(\%)$ & - & $1(0.9)$ & - \\
\hline Minor complications, $n(\%)$ & $1(3.2)$ & $3(2.7)$ & 0.75 \\
\hline $\begin{array}{l}\text { Patients with AF recurrence within } \\
12 \text { months, } n \text { (\%) }\end{array}$ & $5(16.1)$ & $41(36.6)$ & $0.031^{*}$ \\
\hline
\end{tabular}

Table III. Laboratory parameters, haemodynamics and sedation-related parameters of patients in both groups

\begin{tabular}{|c|c|c|c|}
\hline Parameter & $\begin{array}{l}\text { Contact force } \\
(n=31)\end{array}$ & $\begin{array}{l}\text { Standard mapping } \\
\qquad(n=112)\end{array}$ & Value of $p$ \\
\hline Baseline systolic blood pressure, mean $\pm \mathrm{SD}$ [mm Hg] & $128.1 \pm 20.1$ & $122.2 \pm 18$ & 0.18 \\
\hline Baseline diastolic blood pressure, mean $\pm \mathrm{SD}[\mathrm{mm} \mathrm{Hg}]$ & $71.6 \pm 14.2$ & $74.8 \pm 11.3$ & 0.25 \\
\hline Post-procedure systolic blood pressure, mean $\pm \mathrm{SD}[\mathrm{mm} \mathrm{Hg}]$ & $108.1 \pm 19.6$ & $104.5 \pm 14.1$ & 0.44 \\
\hline Post-procedure diastolic blood pressure, mean $\pm \mathrm{SD}[\mathrm{mm} \mathrm{Hg}]$ & $68.8 \pm 15$ & $69.1 \pm 9.3$ & 0.95 \\
\hline Baseline $\mathrm{SaO}_{2}$, mean $\pm \mathrm{SD}(\%)$ & $97.6 \pm 2.2$ & $97 \pm 2.1$ & 0.2 \\
\hline Post-procedure $\mathrm{SaO}_{2}$, mean $\pm \mathrm{SD}(\%)$ & $97.1 \pm 2.3$ & $96.1 \pm 2.7$ & 0.12 \\
\hline Haemoglobin, mean \pm SD $[\mathrm{g} / \mathrm{dl}]$ & $13.1 \pm 2$ & $14.1 \pm 2.5$ & 0.14 \\
\hline Glucose, mean \pm SD [mg/dl] & $114.2 \pm 22.9$ & $111.5 \pm 19.9$ & 0.59 \\
\hline Baseline CRP, mean \pm SD [mg/l] & $12.9 \pm 16.1$ & $17.8 \pm 26.3$ & 0.43 \\
\hline Baseline AST, mean \pm SD [U/I] & $37.4 \pm 27$ & $32.3 \pm 17$ & 0.53 \\
\hline Baseline TSH, mean \pm SD [mU/l] & $1.4 \pm 1.3$ & $1.6 \pm 1$ & 0.62 \\
\hline Total midazolam dose, mean \pm SD $[\mathrm{mg}]$ & $6.1 \pm 2$ & $6.4 \pm 2.8$ & 0.69 \\
\hline Total propofol dose, mean \pm SD $[\mathrm{mg}]$ & $937.8 \pm 396.3$ & $954.9 \pm 377.5$ & 0.87 \\
\hline
\end{tabular}

AST - aspartate aminotransferase, $\mathrm{CRP}-\mathrm{C}$-reactive protein, $\mathrm{SaO}_{2}$ - arterial oxygen saturation, TSH - thyroid-stimulating hormone; *statistically significant

the groups (Table III). Recovery from anaesthesia was observed in less than $60 \mathrm{~min}$ in all cases.

Recurrence of $\mathrm{AF}$ at the 12-month follow-up was detected in 5 patients $(16.1 \%)$ in the contact force group, and in 41 (36.6\%) in the control group $(p=0.031)$ (Table II).

Non-scheduled visits occurred in $3(9.7 \%)$ patients in the contact mapping group and in 20 $(17.9 \%)$ patients in the control group $(p=0.27)$. Non-scheduled visits and change of medication during the 12 months of follow-up are listed in Table IV.

\section{Discussion}

Our study compares procedural characteristics and 12-month follow-up data from PVI procedures that were performed with the use of contact force information from the TactiCath, and from procedures without such information.
The duration of procedures with the use of contact force mapping was significantly shorter when compared to standard mapping procedures. This is in accordance with a previously published study on the use of contact force information [10], but in contrast with another study on the efficacy of acute PVI with contact information [16]. Indeed, the use of additional information during established procedures may in general lead to longer procedure duration. On the other hand, the use of contact force information has been shown to increase efficacy of PVI and therefore an endpoint of procedures could be reached earlier, resulting in shorter procedure duration. Care should be taken when interpreting such a result, since comparability of the studies is limited. Gaspar et al. used a design where acute PVI with and without catheter contact information was compared between (randomly assigned) left and right sided PVs of the 
Table IV. Non-scheduled visits and additional medication during 12-month follow-up

\begin{tabular}{|c|c|c|c|}
\hline Variable & $\begin{array}{l}\text { Contact force } \\
\quad(n=31)\end{array}$ & $\begin{array}{l}\text { Standard mapping } \\
(n=112)\end{array}$ & Value of $p$ \\
\hline Total number of patients with unscheduled visits, $n(\%)$ & $3(9.7)$ & $20(17.9)$ & 0.27 \\
\hline \multicolumn{4}{|l|}{ Reason, $n(\%)$ : } \\
\hline AF episode & $2(6.5)$ & $15(13.4)$ & 0.29 \\
\hline Infection & $0(0)$ & $1(0.9)$ & 0.6 \\
\hline Hypertension & $0(0)$ & $2(1.8)$ & 0.45 \\
\hline ACS & $0(0)$ & $1(0.9)$ & 0.6 \\
\hline NSCP & $1(3.2)$ & $1(0.9)$ & 0.33 \\
\hline Hyper-/hypoglycaemia & - & - & - \\
\hline Delayed femoral haematoma & $0(0)$ & $1(0.9)$ & 0.6 \\
\hline \multicolumn{4}{|l|}{ Addition of drugs during 12 months post ablation, $n(\%)$ : } \\
\hline Amiodarone & $0(0)$ & $1(0.9)$ & 0.6 \\
\hline$\beta$-Blocker & $1(3.2)$ & $0(0)$ & 0.56 \\
\hline Digitoxin & $2(6.5)$ & $1(0.9)$ & 0.056 \\
\hline ACEI & $2(6.5)$ & $2(1.8)$ & 0.16 \\
\hline ARB & - & - & - \\
\hline $\mathrm{CCB}$ & - & - & - \\
\hline Statin & $0(0)$ & $1(0.9)$ & 0.6 \\
\hline Diuretics & - & - & - \\
\hline Aspirin & - & - & - \\
\hline Oral anti-diabetics & $0(0)$ & $2(1.8)$ & 0.45 \\
\hline Insulin & $0(0)$ & $2(1.8)$ & 0.45 \\
\hline Anti-obstructives & $1(3.2)$ & $0(0)$ & 0.56 \\
\hline
\end{tabular}

$A C E I$ - angiotensin-converting enzyme inhibitor, ACS - acute coronary syndrome, AF-atrial fibrillation, ARB - angiotensin receptor blocker, CCB - calcium channel blocker, NSCP - non-specific chest pain (chest paint not related to ACS, pericardial effusion/pericarditis or pneumonia)

same patient [16]. Those authors report a slightly longer duration (26.5 min vs. $23.5 \mathrm{~min}$ ) of the PVIs performed with contact information [16]. A different approach was used in the study by Gaspar et al. compared to our study with a different design, different ablation catheters and different techniques for obtaining catheter contact information $[10,16]$. The strength of their study is that ablation with contact information was performed and compared with conventional ablation in the same patient, so that confounding factors such as degree of fibrosis, LA diameter, type of AF and medication (which may be distributed differently in two study collectives) were ruled out. The procedure duration is most likely a consequence of the study design with its relatively extensive and complex mapping approach. To compare procedural duration of different catheter approaches in two collectives was not an endpoint of their study [16].

There were no significant differences in the number of cases where acute PVI was achieved. No steam pops were recognized. One case of pericardial effusion occurred in the standard mapping group, and this was successfully treated with pericardial puncture and drainage.
Follow-up data show a significantly lower rate of recurrence of AF after 12 months of follow-up in the contact mapping group. The results support the hypothesis of an increase of efficacy and sustainability of RF lesions that are applied with the use of contact monitoring, although the study groups are too small to confirm efficacy and safety of this approach. Our results are in line with previously reported data: ablation lines performed with the guidance of catheter-tissue contact information show fewer gaps [16] and ablation with contact monitoring results in a lower rate of acute PV reconnections [17]. Therefore, an effect on midterm and long-term success rates of PVI procedures may be assumed.

In our study collective, there was a trend to a higher percentage of patients with paroxysmal AF in the control group, whereas the LVEF tended to be higher in the contact force group (Table I). Although not statistically significant, both parameters my have an influence on incidence and treatment response of AF [1, 2]. Persistent AF is usually more complex and associated with a higher recurrence rate after ablation [1, 2]. A higher percentage of patients with persistent $A F-$ as in the contact 
force group in our study - could lead to a reduced success rate of ablation procedures. Furthermore, heart failure predisposes to AF and therefore a reduced AF may be associated with worse outcomes after AF ablation, which could influence our study results. Of note, the mean LVEF was well above $50 \%$ in both groups ( $56.8 \pm 4.9 \%$ vs. $55.6 \pm 3.1 \%)$ and the incidence of structural heart disease did not differ significantly between the groups $(p=0.78$, Table I). Yet, an influence of both parameters on ablation success cannot be concluded from our study and future studies are needed to clarify this issue.

Diabetes mellitus [18] and inflammation [19] have been shown to influence AF and AF recurrence. There were no significant differences in the number of patients with diabetes mellitus or with infections during the follow-up between the two study groups.

The increased success rate in the TactiCath group in our study may be due to several reasons. As it has been discussed before, during conventional AF ablation procedures the amplitude of local atrial electrograms is the main parameter in the evaluation of catheter-tissue contact $[16,17$, 20]. Furthermore, the reduction of local electrograms during ablation is considered to be a sign of successful ablation. When no CF information is provided it can be difficult to decide whether a local electrogram with low amplitude is a result of poor catheter-tissue contact or of fibrotic myocardium. Other parameters such as tactile feedback or fluoroscopic orientation of the catheter tip are subjective and interference-prone [17, 20]. Without the additional information of CF measurement the reduction of local electrograms often may only be a result of catheter instability, especially since respiration and myocardial contraction have been shown to reduce catheter stability and CF [21, 22].

Additionally, it has been postulated that local oedema and acute injury of myocardial cells can lead to electrical stunning with an intraprocedural conduction block, even though no sustainable lesion develops [23]. This could in particular be the case in atrial areas that are difficult to reach with the catheter [6]. The endpoint of a (short-term) conduction block could therefore be misleading without the CF information. In fact, it has been shown that catheter-tissue contact varies between different anatomical sites [6].

Clearly, both catheter instability due to respiration and contraction and the fact that certain anatomical areas are difficult to reach may have a negative impact on lesion formation. Smaller or superficial lesions and gaps between the lesions may result in an increased risk of $\mathrm{PV}$ reconnection and AF recurrence. Improved lesion formation due to catheter-tissue contact monitoring may be the explanation for reduced recurrence rates in patients ablated with the use of CF information.

There are limitations of the study, which should be acknowledged. Besides the relatively small number of patients in our pilot study, we used a non-randomized observational study protocol. A future randomized trial in a larger study population is needed to confirm our results.

In conclusion, we present the first pilot data indicating that contact force information from the TactiCath during PVI results in a significantly shorter procedure duration and a significantly lower rate of recurrence of AF after 12 months, when compared to standard PVI without this information. Our results highlight the impact of contact force information for ablation procedures.

\section{References}

1. Camm AJ, Lip GY, De Caterina R, et al. 2012 focused update of the ESC Guidelines for the management of atrial fibrillation: an update of the 2010 ESC Guidelines for the management of atrial fibrillation. Developed with the special contribution of the European Heart Rhythm Association. Eur Heart J 2012; 33: 2719-47.

2. Calkins H, Kuck KH, Cappato R, et al. 2012 HRS/EHRA/ ECAS expert consensus statement on catheter and surgical ablation of atrial fibrillation: recommendations for patient selection, procedural techniques, patient management and follow-up, definitions, endpoints, and research trial design. J Interv Cardiac Electrophysiol 2012; 33: 171-257.

3. Ouyang F, Antz M, Ernst S, et al. Recovered pulmonary vein conduction as a dominant factor for recurrent atrial tachyarrhythmias after complete circular isolation of the PVs: lessons from double Lasso technique. Circulation 2005; 111: 127-35.

4. Yokoyama K, Nakagawa H, Shah DC, et al. Novel contact force sensor incorporated in irrigated radiofrequency ablation catheter predicts lesion size and incidence of steam pop and thrombus. Circ Arrhythm Electrophysiol 2008; 1: 354-62.

5. Kuck KH, Reddy VY, Schmidt B, et al. A novel radiofrequency ablation catheter using contact force sensing: Toccata study. Heart Rhythm 2012; 9: 18-23.

6. Kumar S, Morton JB, Lee J, et al. Prospective characterization of catheter-tissue contact force at different anatomic sites during antral pulmonary vein isolation. Circ Arrhythm Electrophysiol 2012; 5: 1124-9.

7. Thiagalingam A, D'Avila A, Foley L, et al. Importance of catheter contact force during irrigated radiofrequency ablation: evaluation in a porcine ex vivo model using a force-sensing catheter. J Cardiovasc Electrophysiol 2010; 21: 806-11.

8. Shah D, Lambert H, Langenkamp A, et al. Catheter tip force required for mechanical perforation of porcine cardiac chambers. Europace 2011; 13: 277-83.

9. Wittkampf FHM, Nakagawa H. RF catheter ablation: lessons and lesions. Pacing Clin Electrophysiol 2006; 29: 1285-97.

10. Martinek M, Lemes C, Sigmund E, et al. Clinical impact of an open-irrigated radiofrequency catheter with direct force measurement on atrial fibrillation ablation. Pacing Clin Electrophysiol 2012; 35: 1312-8. 
11. Piorkowski C, Sih $\mathrm{H}$, Sommer P, et al. First in human validation of impedance-based catheter tip-to-tissue contact assessment in the left atrium. J Cardiovasc Electrophysiol 2009; 20: 1366-73.

12. Reddy VY, Neuzil P, Kautzner J, et al. Low catheter-tissue contact force results in late pv reconnection - initial results from EFFICAS I. Heart Rhythm 2011; 8: S26.

13. Shah DC, Reddy VY, Kautzner J, et al. Contact force during ablation predicts af recurrence at 12 months. Heart Rhythm 2011; 8: S447-8.

14. Maagh P, Butz T, Plehn G, Christoph A, Meissner A. Pulmonary vein isolation in 2012: is it necessary to perform a time consuming electrophysical mapping or should we focus on rapid and safe therapies? A retrospective analysis of different ablation tools. Int J Med Sci 2013; 10: 24-33.

15. Wutzler A, Rolf S, Huemer M, et al. Safety aspects of deep sedation during catheter ablation of atrial fibrillation. Pacing Clin Electrophysiol 2012; 35: 38-43.

16. Gaspar T, Sih H, Hindricks G, et al. Use of electrical coupling information in AF catheter ablation: a prospective randomized pilot study. Heart Rhythm 2013; 10: 176-81.

17. Haldar S, Jarman JW, Panikker S, et al. Contact force sensing technology identifies sites of inadequate contact and reduces acute pulmonary vein reconnection: a prospective case control study. Int J Cardiol 2013; 168: 1160-6.

18. Bissinger A, Grycewicz T, Grabowicz W, Lubinski A. The effect of diabetic autonomic neuropathy on P-wave duration, dispersion and atrial fibrillation. Arch Med Sci 2011; 7: 806-12.

19. Lin Q, Jia L, Sun Y. A pilot study of circulating PPAR- receptor protein in elderly patients with atrial fibrillation. Arch Med Sci 2012; 8: 471-6.

20. Holmes D, Fish JM, Byrd A, et al. Contact sensing provides a highly accurate means to titrate radiofrequency ablation lesion depth. J Cardiovasc Electrophysiol 2011; 22: 684-90.

21. Reddy VY, Shah D, Kautzner J, et al. The relationship between contact force and clinical outcome during radiofrequency catheter ablation of atrial fibrillation in the TOCCATA study. Heart Rhythm 2012; 9: 1789-95.

22. Kumar S, Morton JB, Halloran K, et al. Effect of respiration on catheter-tissue contact force during ablation of atrial arrhythmias. Heart Rhythm 2012; 9: 1041-7.

23. Miller MA, d'Avila A, Dukkipati SR, et al. Acute electrical isolation is a necessary but insufficient endpoint for achieving durable PV isolation: the importance of closing the visual gap. Europace 2012; 14: 653-60. 